\title{
The Alarming Toxicity of Ruta Graveolens
}

\author{
Moges Abebe* \\ Department of Biological and Physical Sciences, Saint Augustine University, USA
}

*Corresponding author: Moges Abebe, Department of Biological and Physical Sciences, Saint Augustine University, USA

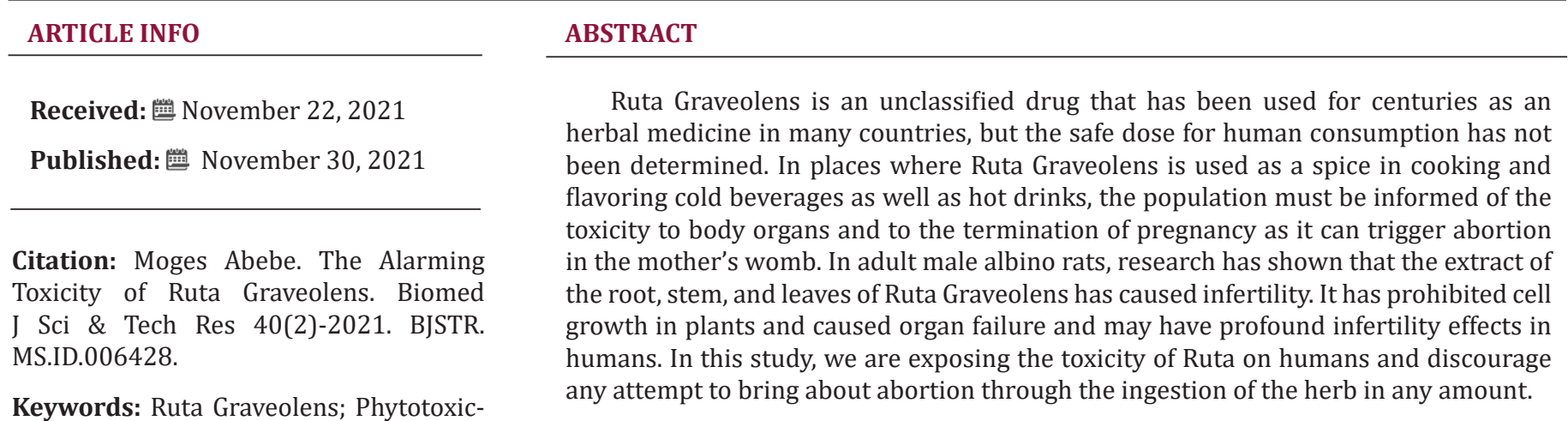
ity

\section{Introduction}

Worldwide use of Ruta Graveolens as an herb and as alternative medicine has increased dramatically in the past decades without any increased effort to compensate for the toxicity and the unsafe human consumption. While pharmacological studies on drugs that cause cardiac, hepatic, and nephrotoxicity have resulted in drug termination, the same type of studies on herbal medicine are ignored and actions have not been taken to protect the youth from this herb in their productive stages of life. The endemic production of Ruta Graveolens is in India [1], Ethiopia [2], and South Africa [3] where there is a dramatic growth in population that masks the toxic and infertility effect on the younger population. The purpose of this study is to bring awareness that Ruta Graveolens is a toxic plant and should be handled with caution.

Scholarly articles have reported their findings on the abortifacient properties of herbs resulting in miscarriages followed by a likely chance of organ failure, especially kidney failure in pregnant mothers. In herbal medicine, Ruta is an emmenagogues herb that is taken by women to induce and increase menstrual flows and to cleanse the body. It is reported that Ruta has been used as an abortifacient in Europe, Asia, Africa, and South America and is found to be endemic in the Mediterranean countries. Studies over the past twenty-one years on both female and male mammals have shown abortive effects, specifically implantation, premature infant losses, and paralyzing sperm activities. The chemical composition analysis of Ruta Graveolens listed 50-120 different compounds among which Kuzovkina in his 2004 publication found coumarins and xanthotoxins as the toxic infertility drugs known to man. The most active component of Ruta Graveolens extract is chalepensin, widely known as an anti-fertility drug in mammals. In cultural medicine, Ruta Graveolens is still used as an anti-inflammation, anticancer, antioxidant, antidiabetic, antibacterial, and antifungal medicine. Figure 1 shows a picture of Ruta Graveolens grown in our laboratory. 


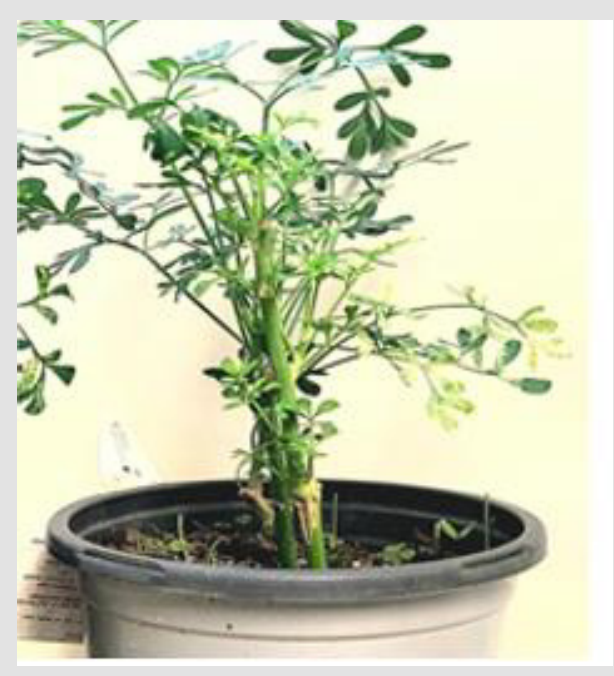

Figure 1: Ruta Graveolens.

\section{Methodology}

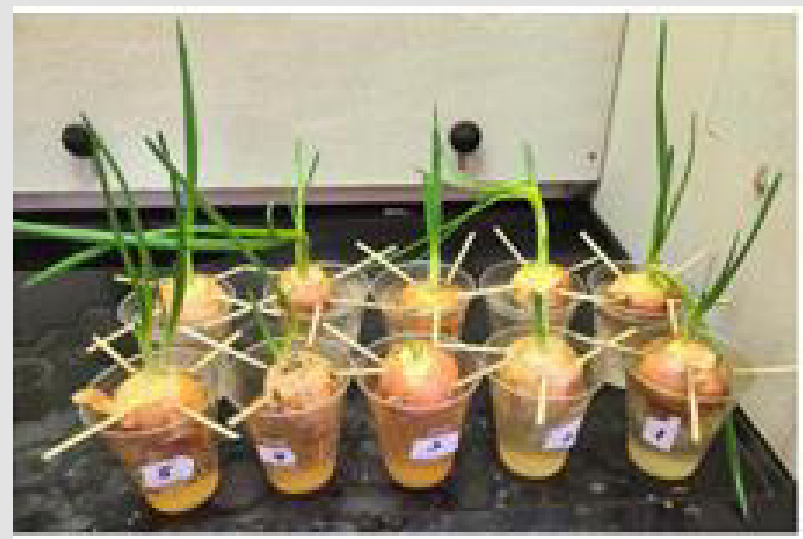

Figure 2: Treated Onion bulbs with Rue.

In this study, Ruta was extracted by diethyl ether, hydrochloric acid, and sodium hydroxide solutions and was used on the commercially purchased organic onions and the growth rate of onion roots was observed. Rationally, we used onion roots to avoid animal sacrifice. The three extracts were administered in the solution where the onions were grown in the amount of 1,2 , 5, 10, 15, 25 milliliters and the growth rate were measured. Pure water was used in the control sample. A review of literature lists several methods of extraction of phytochemical compounds like coumarins and xanthotoxins which is effectively done with a hot water extract and or a variety of pure and mixed organic solvents of ethanol, hexane, chloroform, acetone, and a combination of these solvents. Separation of the essential oil and other chemicals from the stem, leaves, roots, and seeds of Ruta was done by several planned extraction steps and the results were successful. Figure 2 is the setup of the onion bulbs hanging on sticks and the roots were submerged in water. The submersion water was replenished every day and onions were exposed to open air sunlight. At the end of 15 days, the roots and stems were shaved, dried, and weighed along with the control group.

The following research approach is reviewed below to show that the extraction of Ruta Graveolens by different solvents and different testing methodology compliments the method we chose in this study. Kong et al [4]. performed solvent extraction of $10.4 \mathrm{Kg}$ of powdered whole plant of Ruta Graveolens with petroleum ether and chloroform that percolated overnight. The extracts from the root, stem, and leaves were separated into its components using benzene and methanol. They succeeded in isolating chalepensin, a toxic compound among furanocoumarin which is a pharmacologically active compound in Ruta Graveolens. They purified chalepensin and the yield was 9 grams of the crystal that was tested and confirmed with proton NMR and mass spectroscopy. The effects of infertility due to Ruta Graveolens were tested on 10 Sprague Dawley rats that were randomly selected from a large selection of genetically identical 8 weeks old females. The pregnant rats were kept in an environmentally controlled room, with minimized infections, and a supply of nutrition. Solvent enhanced chalepensin extract was administered to the experimental group and the control group.

Hale et al [5]. studied Ruta Graveolens leaves, seeds, flowers, and stems obtained from credible sources and separated for extraction. The sample was blended, the extraction was conducted by several volatile liquids, and the isolated crystals were separated into fractions and tested using TLC and proton and carbon 13 NMR, and UV-VIS spectroscopic methods. Fresh leaf extraction was purified and isolated using several steps in the extraction process. All the extracts from their experiment inhibited cell division in the root tip of duckweed. Gonçalves de Freitas [6]. studied Ruta Graveolens grown in Brazil. These were air-dried, grounded, and extracted using $70 \%$ ethanol with a $25 \%$ yield. The selected CF1 mice were supervised in a controlled environment with nutritional supplies and an environmentally controlled laboratory. They administered $1000 \mathrm{mg} / \mathrm{kg}$ of extract per body weight to the experimental group and only the vehicle was administered to the control group. The extract was administered to the 10 mice per group at different stages of pregnancy. The mice were sacrificed and checked for the condition of the fetus in their ovaries.

Indian [1] grown Ruta Graveolens was collected, and aerial parts and the roots were separately, dried, and powdered, and made as a paste with water. The plant gave a $20 \%$ slurry extract, and the paste was administered to rats and hamsters. The ingestion dose was $8 \mathrm{~g} / \mathrm{kg}$, and it was administered to rats and hamsters within two weeks of pregnancy. Then healthy female rats and hamsters were used in a controlled environment and experimental and controlled groups were used. At the end of the experimental comparison of body weight, before and after the trial was used, 
the effect of the toxicity of the Ruta Graveolens on the inner body organs was investigated. Statistical significance using $(\mathrm{P}, 0.05)$ chi-square analysis was obtained. The results showed increased toxicity and a high rate of fetus mortality. The chloroform extract chalepnisin was found in all parts of the plant, and its toxicity was reported in the pregnant rodents. The results showed a significant increase in infertility effect in the pregnant rats, and the experiment revealed chalepnisin, as the specific compound that has a significant effect on fertility. Finally, Ruta Graveolens leaves grown in Peru [7] were stove dried, pulverized into powder, and produced the collected extract using water as a solvent. The extracted liquid was evaporated and administered as $10 \mathrm{~mL}$ injection per mouse. Most of these methods that are published in the literature used rodents and animals' specimen for their study investigation, and our experimental study of the toxicity of Ruta on plants is a good complement to the collection.

\section{Results and Discussion}

This study showed significant effect in the retardation of onion root cell growth in our laboratory. Onion root growth that was treated with different concentrations of ether extracts were graphed in Figure 3. The ruta extract showed its growth inhibitory effect in all three extracts. All the variables like the $\mathrm{pH}(6.0+0.5)$, temperature $\left(23+1^{\circ} \mathrm{C}\right)$, and atmospheric pressure were the same for the control group and the experiment. The experiment was stopped after 15 days, the roots and stem were shaved from the onion bulb, and the dried sample was weighed for the 1-10 mL ether extract solution of Ruta Graveolens. The onion bulbs died when any significant Ruta Graveolens extract was added to the water where the plant was partially immersed. In other studies, the toxicity of the Ruta extract has also prohibited other researchers from coming to a firm conclusion Y. C. Kong et al. [4] did not come to a clear conclusion in the Ruta's anti-infertility and abortion effect, and their results didn't produce any clear-cut conclusion. The amount of Ruta Graveolens they used in rats was far too toxic to the specimen and organ failure resulted before the infertility rate was observed. The chlorophyll-water extract they used was less toxic, but the cumrain and specifically chalepensin's anti-implantation and infertility has not been proven.

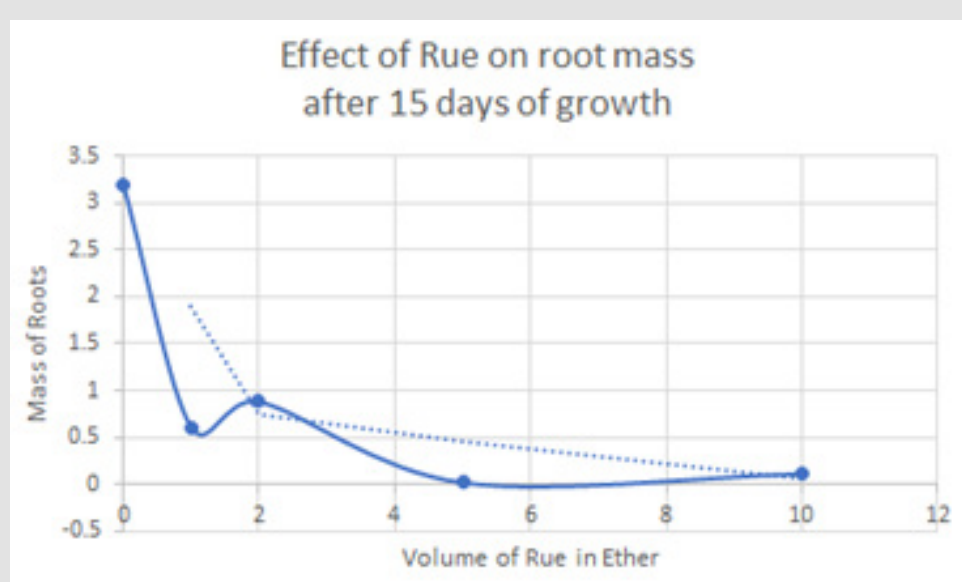

Figure 3: Root Growth of onion bulb after addition of different volumes of Rue extract by ether.

Anderson Gonçalves de Freitas et al. [6] also found that Ruta Graveolens did not result in infertility and anti-implantation in pregnant mouse. However, their results showed good evidence of fetotoxicity after the seventh DOP pregnant mouse that received the herbal extract. The use of Ruta Graveolens resulted in organ failure, and they concluded that the herb should not be used in humans as a medicinal herb or as a contraceptive. Manoj Gandhi et al. studied the effect of Ruta Graveolens in rats and hamsters for infertility activities. They used methanol and petroleum ether extracts and found that the herb caused acute toxicity in both the rats and hamsters used. In their study, the toxicity effect interfered with the result. Gutiérrez-Pajares et al. [7] exposed pregnant mice to Ruta Graveolens during the first four days of pregnancy. The super ovulated pregnant mice different amounts of the aqueous extract. The administration of Ruta Graveolens to goats was found to attack the kidneys and higher doses were toxic resulting in death. The oil extract from the leaves has the potential toxicity that will result in uterine hemorrhaging. They were cautious to advise that their finding in animals cannot be transferred to humans. Their studies showed that ingested Ruta Graveolens is toxic enough to create an unfavorable environment for embryo implantation and will also retard fetal growth at any stage of fetus development. They isolated the alkaloid components of the herb like flavonoids, acridones, and furanocoumarins that are known growth inhibitors, 
and they concluded that the herb reduces implantation and growth development in mice. In our experiment, the toxicity level that will kill the onion was determined to be $5 \mathrm{~mL}$ of ether extract of Ruta, and we used 1-4 mL of volumes to gather our conclusions. Figure 3 showed $99.4 \%$ retardation of onion root growth while 1- and 2-mL extract resulted in $72.4 \%$ and $80.8 \%$ respective retardation $[8,9]$.

\section{Conclusion}

The use of Ruta Graveolens, an unclassified drug, reported in this paper shows that the dosage level used for experimentation can very easily reach the toxic level before recognizing its anti-cell growth characteristics. We were successful to show the growth retardation of Ruta Graveolens in onion bulbs by plant-based research and applying the toxicity of a plant against another plant. The growth rate inhibition may be the root cause of the herb's damage to unborn babies. In the studies reviewed, the toxicity of the chalepension which is extracted from the herb, led to organ failure and death in rats before any meaningful infertility result was achieved. It will be interesting to see further studies to implement Ruta Graveolens as an organically grown safe and herbal alternative to currently available hormone contraception. Ruta Graveolens is shown here as a plant-growth inhibitor, and this property of the herb may open the door for the development of future organic herbicide development. Furthermore, Ruta's cell growth inhibition has been studied in anticancer melanoma, and it will be beneficial

ISSN: 2574-1241

DOI: 10.26717/BJSTR.2021.40.006428

Moges Abebe. Biomed J Sci \& Tech Res

cC (P) This work is licensed under Creative

Submission Link: https://biomedres.us/submit-manuscript.php to see further research in implementing Ruta for other medicinal purposes. In conclusion, the toxicity of Ruta Graveolens is alarming, and this study showed that onion cell growth inhibition was demonstrated.

\section{References}

1. Gandhi M, R Lal, A Sankaranarayanan, P L Sharma (1991) Post-Coital Infertility Action of Ruta Graveolens in Female Rats and Hamsters. J Ethnopharmacol 34(1): 49-59.

2. Desta B Ethiopian (1994) Traditional Herbal Drugs Part III. infertility Activity of 70 Medicinal Plants. J Ethnopharmacol 44(3): 199-209.

3. Sethi N, Nath D, Snigh RK, Srivastava RK (1990) Antifertility And Teratogenic Activity of Some Indigenous Medicinal Plants In Rats. Fitoterapia LXI, p. 64-66.

4. Kong Y C, C P Lau, K H Wat, K H Ng, P P H But, et al. (1989) "Infertility Principle of Ruta Graveolens." Planta Med 55(2): 176-178.

5. Hale, Amber L, Kumudini M Meepagala, Anna Oliva, Giovanni Aliotta, et al. (2004) Phytotoxins from the Leaves of Ruta Graveolens. J Agric Food Chem 52(11): 3345-3349.

6. Goncalves de Freitas, Tanise Goncalves de Freitas, Patrice Martins Augusto, Tatiana Montanari (2005) Effect of Ruta Graveolens L on pregnant mice. Contraception 71(1): 74-77.

7. Gutierrez Pajares JL, L Zuniga (2003) Ruta Graveolens Aqueous Extract Retards Mouse Preimplantation Embryo Development. J Pino Reproductive Toxicology - Elsevier 17(6): 667-672.

8. Elisabetsky E, Posey DA (1989) Use of Contraceptive and Related Plants by the Kayapo Indians (Brazil). J Ethnopharmacol 26(3): 299-316.

9. Li P (1988) Preliminary Study on Anti-Implantation and Anti-Early Fertility Effect of Chinese Herbal Medicine. J of XI AN, pp. 317- 320.

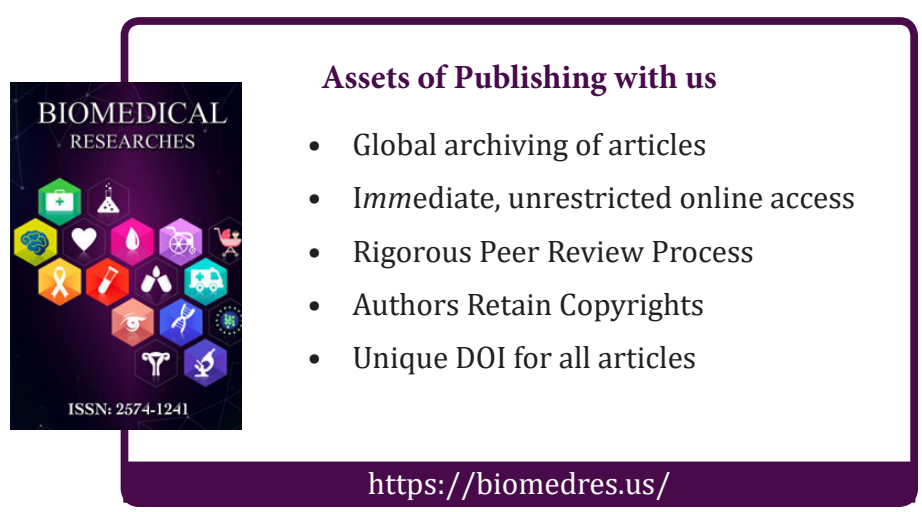

